\title{
Fano Resonance Investigation of PbTe Layers Containing Eu and Gd Ions
}

\author{
B.A. Orlowski ${ }^{a, *}, \mathrm{~V} . \mathrm{Osinniy}^{a}, \mathrm{P} . \mathrm{Dziawa}^{a}, \mathrm{M} \mathrm{PietrzyK}^{a}$, \\ B.J. Kowalski ${ }^{a}$, B. TAliashvili ${ }^{a}$, T. Story ${ }^{a}$ \\ AND R.L. JOHNSON ${ }^{b}$ \\ ${ }^{a}$ Institute of Physics, Polish Academy of Sciences \\ al. Lotników 32/46, 02-668 Warsaw, Poland \\ ${ }^{b}$ Institut für Experimentalphysik, Universität Hamburg \\ Luruper Chaussee 149, 22761 Hamburg, Germany
}

The Fano resonance photoemission studies of $\mathrm{Gd} /(\mathrm{Pb}, \mathrm{Gd})$ Te layers using synchrotron radiation were carried out and the electronic structure parameters like binding energies of $\mathrm{Gd}^{3+} 4 f$ and $5 p$ shells, resonance and antiresonance energies for $\mathrm{Gd}^{3+}$ were determined. The presence of $\mathrm{Eu}^{3+}$ ions was observed in the $(\mathrm{Pb}, \mathrm{Eu}) \mathrm{Te}$ and $(\mathrm{Eu}, \mathrm{Gd}) \mathrm{Te}$ layers grown by MBE technique. The comparison of data for $(\mathrm{Pb}, \mathrm{Gd}) \mathrm{Te}$ compound with corresponding data for $(\mathrm{Eu}, \mathrm{Gd}) \mathrm{Te}$ and $(\mathrm{Pb}, \mathrm{Eu})$ Te layers indicates that we are not able to distinct the $\mathrm{Eu}^{3+} 4 f$ and $\mathrm{Gd}^{3+} 4 f$ electrons contribution to the valence band photoemission spectra because of small content od Gd and similar binding energy values. The key parameters allowing to prove exactly the presence of either $\mathrm{Eu}^{3+}$ or $\mathrm{Gd}^{3+}$ are the resonance and antiresonance energies which are significantly different for these ions and equal to $143 \mathrm{eV} / 137 \mathrm{eV}$ and $150 \mathrm{eV} / 142 \mathrm{eV}$, respectively.

PACS numbers: 79.60.-i, 71.20.Mq

\section{Introduction}

The narrow gap IV-VI semiconductors like PbTe attract remarkable interest because of its wide application as a material for infrared lasers, infrared detectors and thermoelectric devices [1]. The PbTe crystallizes in the rock salt structure and in the same structure crystallizes EuTe - a wide band gap ionic Heisenberg magnetic semiconductor [2]. Both crystals are the good partners for creation of ternary alloys and for heteroepitaxy. The substitution of $\mathrm{Gd}^{3+}$ for $\mathrm{Eu}^{2+}$ leads to the introduction of Gd $5 d$ electrons as donors, leading to the increase in electrical conductance of EuTe. Appearance of free carriers leads to the replacement of antiferromagnetic order of EuTe layer by ferromagnetic state due to Ruderman-

*corresponding author 
Kittel-Kasuya-Yosida (RKKY) interaction. The presented paper concerns the study of influence of technological parameters on growing these materials and of contribution of $4 f$ electrons to the created material electronic structure.

\section{Resonance photoemission and synchrotron radiation}

The effect of electron photoemission appears when a sample is irradiated with photons of energy sufficient to photoemit electrons from the sample. When the photons beam is monochromatic we can use electron energy analyzer to measure the energy distribution curve (EDC) of photoemitted electrons. The structure of EDC is carrying information about the crystal electronic structure. Changing the $h \nu$ energy we can reach the region of absorption edge of atom (component of the crystal) and to excite the atom selectively and locally. In this case the resonance arises due to quantum mechanical interference between the direct photoemission process and a super Coster-Kronig decay of excited atom (Fano effect [3]). In the case of rare earth elements the interference appears between direct photoemission process and e.g. excitation $4 d-4 f$ of Gd ion at $h \nu$ energy of about $150 \mathrm{eV}$ (Gd absorption edge region). Both effects can interfere

$$
\mathrm{RE} 4 d^{10} 4 f^{7}+h \nu \rightarrow \mathrm{RE} 4 d^{10} 4 f^{6}+\mathrm{e}^{-}-\text {direct photoemission process }
$$

and super Coster-Kronig decay

$$
\mathrm{RE} 4 d^{10} 4 f^{7}+h \nu \rightarrow\left[\mathrm{RE} 4 d^{9} 4 f^{8}\right](\text { excited state }) \rightarrow \mathrm{RE} 4 d^{10} 4 f^{6}+\mathrm{e}^{-} .
$$

Quantum mechanical interference occurs between these two types of processes involving the same initial and final states, thereby enhancing or damping the electron emission when $h \nu$ is in the resonance or antiresonance region, respectively. The resulting photon energy dependent intensity profile can be described by the Fano interference formalism [3]. To perform the experiment of resonant photoemission spectroscopy the synchrotron radiation is a perfect source of excitation [4-6]. It allows producing intense and collimated light with needed wide range of $h \nu$ energy.

\section{Experiments and discussion}

The layers were grown in MBE machine using effusion cells for $\mathrm{PbTe}, \mathrm{Eu}$, $\mathrm{Gd}$, and Te. The crystal of $\mathrm{BaF}_{2}$ (111) surface (rock salt structure, lattice constant $a_{0}=6.20 \AA$ ) freshly cleaved in air and annealed in $560^{\circ} \mathrm{C}$ in $\mathrm{UHV} \mathrm{MBE}$ chamber was used as substrate for deposition. The buffer layer of $\mathrm{PbTe}$ of about 20 to $600 \mathrm{~nm}$ thickness was deposited at substrate temperature of $270^{\circ} \mathrm{C}$. The layers were deposited on top of buffer layer at the same substrate temperature. The two-dimensional growth was monitored by electron diffraction (RHEED) during process performance. The roughness of surface was studied by atomic force microscopy (AFM) technique. The Gd concentration determined from X-ray fluorescence (XRF) data and independently from the Vegard law was equal to 2 at.\% [7]. The details about MBE layers technology and first characterization are presented in Ref. [8]. The cleaning of the surface (etching by Ar ion $600 \mathrm{eV}$ energy and annealing in $300^{\circ} \mathrm{C}$ ), atoms $\mathrm{Eu}$ and $\mathrm{Gd}$ deposition and photoemission 
measurements presented in the paper were performed at the FLIPPER II station of synchrotron storage ring in HASYLAB, DESY (Germany) [9-12].

The paper concerns to the set of selected results of electronic structure study of narrow gap semiconductor crystal like PbTe heavily doped with rare earth atoms $\mathrm{Eu}$ and Gd and to the rare earth telluride's semiconductor thin layers investigation done using resonant photoemission spectroscopy. The previous studies of $(\mathrm{Eu}, \mathrm{Gd}) \mathrm{Te}$ thin layers [12] showed the presence of $\mathrm{Eu}^{3+}$ ions at the surface of layer and dominant presence of $\mathrm{Eu}^{2+}$ ions inside the layer. Unfortunately, due to the technology limits the maximum amount of Gd ions in this compound cannot overcome 1-2 at.\%. Because $\mathrm{Eu}^{3+} 4 f$ and $\mathrm{Gd}^{3+} 4 f$ electrons have the similar binding energies, we were not able to determine their binding energies for this case and the presence of $\mathrm{Gd}$ ions was possible to detect basing on high energy photoemission spectra for $3 d$ core levels [12]. To increase the atomic percentage of Gd in PbTe the experiment was performed to deposit controlled amount of Gd atoms $(25 \AA$ on $6000 \AA$ A PbTe, Fig. 1) on PbTe (111) clean surface and annealing of the sample. It gives the possibility to compare the binding energy and resonance energy for $\mathrm{Gd}^{3+} 4 f$ electrons in $(\mathrm{Pb}, \mathrm{Gd}) \mathrm{Te}$ as a bulk crystal and in thin layers of PbTe with deposited on it and diffused Gd atoms. The same comparison of binding energy and resonance energy can be done for $\mathrm{Eu}^{3+} 4 f$ electrons in $(\mathrm{Pb}, \mathrm{Eu}) \mathrm{Te}$ thin layers with the layers of PbTe and deposited on it and diffused Eu atoms (see Table).

After Gd deposition, the Gd metallic layer appears on the surface. The annealing of the sample leads to the diffusion of deposited Gd atoms to the substrate. The set of photoemission spectra of valence band of annealed structure for different photon energies is presented in Fig. 1. For increasing $h \nu$ energies the increase in intensity of the Gd $4 f$ peak is observed and maximum value is achieved at photon energy about $150 \mathrm{eV}$. This behavior indicates the resonance character of photoemission process. Based on these data we observed the Gd contribution to the valence band spectra: one maximum of $\mathrm{Gd}^{3+} 4 f$ with binding energy about $9.6 \mathrm{eV}$ and two peaks of $\mathrm{Gd}^{3+} 5 p$ with binding energies $24.4 \mathrm{eV}$ and $29.5 \mathrm{eV}$. The Fano resonance curves for $\mathrm{Gd}^{3+} 4 f$ and $\mathrm{Gd}^{3+} 5 p$ presented in Fig. 2 were obtained basing on the change of area of the corresponding peak of obtained photoemission spectra presented in Fig. 1. From these curves the resonance energy for $\mathrm{Gd}^{3+}$ was found to be equal to about $150 \mathrm{eV}$ and antiresonance energy to be $140 \mathrm{eV}$. In Fig. 3 the Fano resonance curves for $4 f$ shell electron of $\mathrm{Eu}^{2+}, \mathrm{Eu}^{3+}$ and $\mathrm{Gd}^{3+}$ ions in $(\mathrm{Eu}, \mathrm{Gd}) \mathrm{Te}$ and $(\mathrm{Pb}, \mathrm{Gd}) \mathrm{Te}$ structures are presented. The lowest resonance energy is related to $\mathrm{Eu}^{2+}$ ions while the highest to $\mathrm{Gd}^{3+}$ ions. The antiresonance energy for $\mathrm{Eu}^{3+}$ is close to the resonance energy for $\mathrm{Eu}^{2+}$ and antiresonance energy for $\mathrm{Gd}^{3+}$ close to $\mathrm{Eu}^{3+}$. This fact indicates that at resonance energy for chosen ion only intensity of peaks corresponding to this ion shows the significant (resonance) increase in EDC curves of the valence band while the intensity of peaks corresponding to the other ions remains without change. It indicates that the resonances behave like being selective and local for particular ions. 


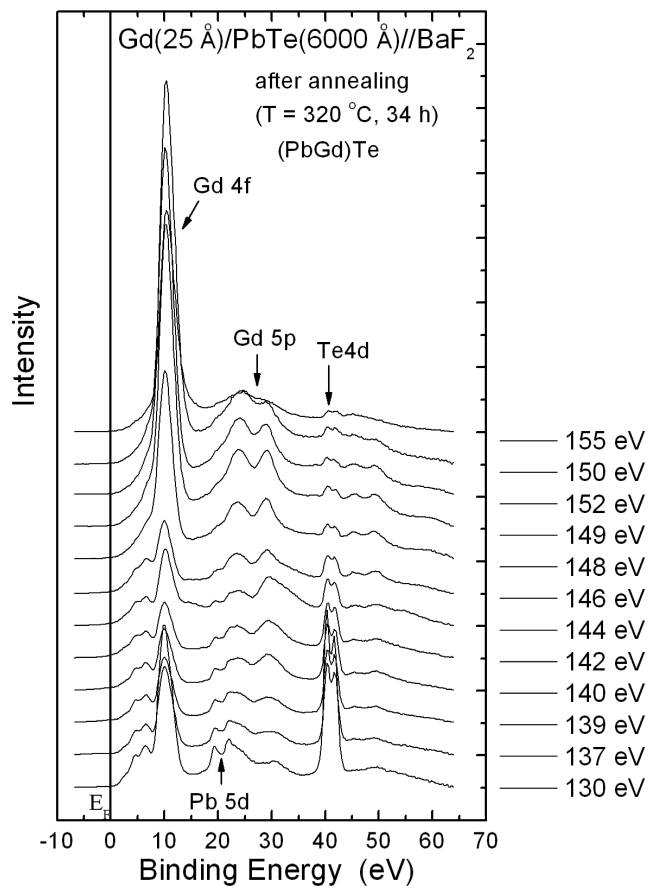

Fig. 1. The set of photoemission spectra of valence band of $\mathrm{Gd} /(\mathrm{Pb}, \mathrm{Gd}) \mathrm{Te}$ layer for different photon energies.

TABLE

Binding energy $\left(E_{\mathrm{B}}\right)$ of $4 f$ electrons of $\mathrm{Eu}^{2+}, \mathrm{Eu}^{3+}$, and $\mathrm{Gd}^{3+}$ ions in $(\mathrm{Pb}, \mathrm{RE}) \mathrm{Te}$ bulk compounds and layers, and photon energies of resonance $\left(h \nu_{\mathrm{res}}\right)$ and antiresonance $\left(h \nu_{\text {ares }}\right)$ for these ions.

\begin{tabular}{c|c|c|c|c|c|c}
\hline \hline \multirow{2}{*}{} & \multicolumn{3}{|c|}{ Thin layers } & \multicolumn{3}{c}{ Bulk crystals } \\
\cline { 2 - 7 } & $E_{\mathrm{B}}[\mathrm{eV}]$ & $h \nu_{\text {res }}[\mathrm{eV}]$ & $h \nu_{\text {ares }}[\mathrm{eV}]$ & $E_{\mathrm{B}}[\mathrm{eV}]$ & $h \nu_{\text {res }}[\mathrm{eV}]$ & $h \nu_{\text {ares }}[\mathrm{eV}]$ \\
\hline $\mathrm{Eu}^{2+}$ & $1.6[13]$ & $140.2[13]$ & $135[13]$ & $2.4[14]$ & $141.4[14]$ & $134.5[14]$ \\
& & $141[10]$ & $132[10]$ & & & \\
\hline $\mathrm{Eu}^{3+}$ & $7.3-10.5[13,10]$ & $143[113]$ & $137[13]$ & & & \\
& & $146[10]$ & $140[10]$ & & & \\
\hline $\mathrm{Gd}^{3+}$ & $10.2[11]$ & $150[11]$ & $142[11]$ & $10[14]$ & $150.4[14]$ & $145[14]$
\end{tabular}

The binding energy, resonance and antiresonance energies for $\mathrm{Eu}^{2+}, \mathrm{Eu}^{3+}$, and $\mathrm{Gd}^{3+}$ ions in the layers and bulk crystal are presented in Table. The absence of $\mathrm{Eu}^{3+}$ ions in bulk of $(\mathrm{Pb}, \mathrm{Eu}) \mathrm{Te}$ crystal is observed. The $\mathrm{Eu}^{3+} 4 f$ shell is characterized by two peaks at $7.3 \mathrm{eV}$ and $10.5 \mathrm{eV}$ while $\mathrm{Gd}^{3+} 4 f$ shell has only one peak at $10.2 \mathrm{eV}$. Taking into account only binding energy data it is difficult to determine if either $\mathrm{Eu}^{3+}$ or $\mathrm{Gd}^{3+}$ ions are present inside PbTe crystal matrix. 


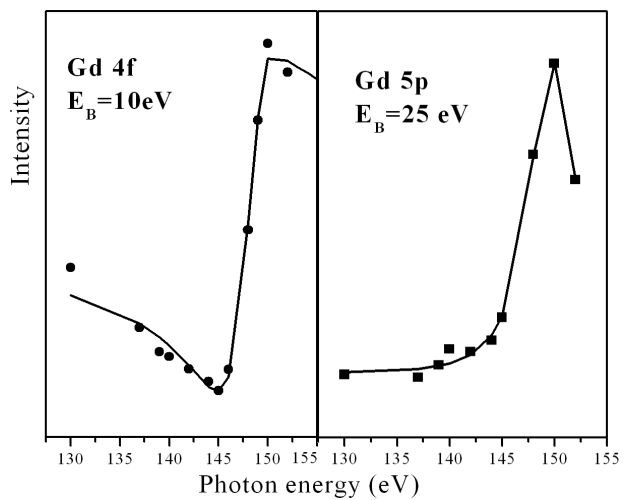

Fig. 2. Fano resonance curves for $\mathrm{Gd}^{3+} 4 f$ and $5 p$ electrons obtained from data presented in Fig. 1.

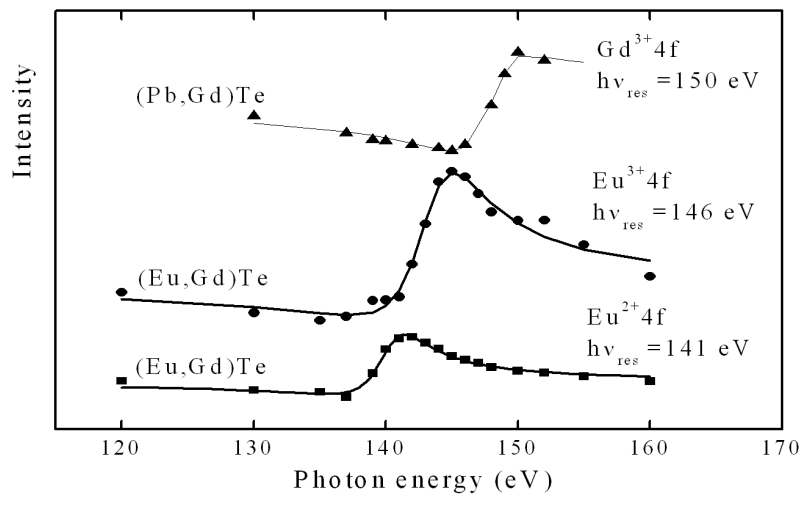

Fig. 3. Fano resonance curves for $\mathrm{Eu}^{2+}, \mathrm{Eu}^{3+}$ in $(\mathrm{Eu}, \mathrm{Gd}) \mathrm{Te}[9]$ and $\mathrm{Gd}^{3+}$ ions in $(\mathrm{Pb}, \mathrm{Gd}) \mathrm{Te}$ layers.

The big difference is observed in the resonance energies which is equal to $7 \mathrm{eV}$. In order to definitively establish which atoms are inside PbTe matrix we need to measure the Fano resonance and determine the resonance energy. The $\mathrm{Gd}^{3+} 4 f$ binding energy and resonance energy in $(\mathrm{Pb}, \mathrm{Gd}) \mathrm{Te}$ thin layers are similar to the values in bulk crystals but of unknown reason the antiresonant energy is lower by $3 \mathrm{eV} . \mathrm{Eu}^{2+} 4 f$ shell is located significantly higher than $\mathrm{Eu}^{3+} 4 f$ and $\mathrm{Gd}^{3+} 4 f$ shell position so in the case when material only consists of $\mathrm{Eu}^{2+}$ and $\mathrm{Gd}^{3+}$ ions. The valence band photoemission spectra will clearly identify the contribution of $\mathrm{Eu}^{2+}$ and $\mathrm{Gd}^{3+}$ ions.

\section{Summary and remarks}

The photoemission studies of (Eu,Gd)Te layers showed that Eu ions are in two charge states: $\mathrm{Eu}^{2+}$ and $\mathrm{Eu}^{3+}$. The structure of $\mathrm{Eu}^{3+} 4 f$ shell located at the valence band is overlapped with $\mathrm{Gd}^{3+} 4 f$ shell. Therefore, for our samples we used 
the method based on the Fano resonance measurements for distinction of $\mathrm{Eu}^{3+}$ $4 f$ and $\mathrm{Gd}^{3+} 4 f$ ions. For this purpose the binding energies and the resonant and antiresonant energies of $\mathrm{Eu}^{3+}$ and $\mathrm{Gd}^{3+}$ ions were determined for $(\mathrm{Pb}, \mathrm{Eu}$ and/or Gd)Te films fabricated by MBE method (Table). After optimization of technology parameters (substrate temperature and rate of evaporation, conditions of surface annealing in UHV) in ( $\mathrm{Pb}, \mathrm{Eu}) \mathrm{Te}$ layers the density of $\mathrm{Eu}^{3+}$ charge states was negligibly small in the volume while at the surface of the layers the amount of $\mathrm{Eu}^{3+}$ ions was increasing after each argon ion bombardment or not proper temperature treatment.

\section{Acknowledgments}

Authors acknowledge support given by MSHE of Poland grants: N 202101 31/0749 and Special Research Project DESY/68/2007.

\section{References}

[1] T. Story, in: Lead Chalcogenides Physics and Applications, Ed. D. Khokhlov, Taylor and Francis, New York 2003, p. 385.

[2] A. Mauger, C. Godart, Phys. Rep. (Rev. Sec. Phys. Lett.) 141, 51 (1986).

[3] U. Fano, Phys. Rev. 124, 1866 (1961).

[4] S. Hufner, Photoelectron Spectroscopy, Springer, New York 1996.

[5] D.W. Lynch, C.G. Olson, Photoemission Studies of High-Temperature Superconductors, Cambridge University Press, Cambridge 1999.

[6] M. Cardona, L. Ley, in: Photoemission in Solids I, Eds. M. Cardona, L. Ley, Springer-Verlag, Berlin 1978, p. 84.

[7] M. Iida, T. Shimizu, H. Enomoto, H. Ozaki, Jpn. J. Appl. Phys. 32, 4449 (1993).

[8] N. Romcevic, A.J. Nadolny, M. Romcevic, T. Story, B. Taliashvili, A. Milutinovic, J. Trajic, E. Lusakowska, D. Vasiljevic-Radovic, V. Domukhovski, V. Osinniy, B. Hadzic, P. Dziawa, J. Alloys Comp. 438, 34 (2007).

[9] B.A. Orlowski, M.A. Pietrzyk, B.J. Kowalski, V. Osinniy, B. Taliashvili, P. Dziawa, T. Story, R.L. Johnson, Proc. HASYLAB-Users Meeting, January 2007, HASYLAB Annual Rep. , 651 (2006).

[10] V. Osinniy, B.A. Orlowski, P. Dziawa, B.J. Kowalski, M. Pietrzyk, B. Taliashvili, S. Mickievicius, T. Story, R.L. Johnson, Bull. Polish Synchr. Rad. Soc. 5, 213 (2006).

[11] M.A. Pietrzyk, B.A. Orlowski, B.J. Kowalski, P. Dziawa, V. Osinniy, B. Taliashvili, R.L. Johnson, Bull. Polish Synchr. Rad. Soc. 5, 204 (2006).

[12] B.A. Orlowski, S. Mickievicius, V. Osinniy, A. Nadolny, B. Taliashvili, P. Dziawa, T. Story, R. Medicherla, W. Drube, Nucl. Instrum. Methods Phys. Res. B 238, 346 (2005).

[13] R. Denecke, L. Ley, G. Springholz, G. Bauer, Phys. Rev. B 53, 4534 (1996).

[14] B.J. Kowalski, Z. Golacki, E. Guziewicz, A. Kozanecki, B.A. Orlowski, J. Ghijsen, R.L. Johnson, J. Alloys Comp. 286, 121 (1999). 